\title{
Mobility as the Purpose of Postural Control
}

\author{
Charlotte Le Mouel * and Romain Brette
}

Institut National de la Santé et de la Recherche Médicale, Centre National de la Recherche Scientifique, Institut de la Vision, Sorbonne Universités, Université Pierre et Marie Curie, Univ. Paris 06, Paris, France

Counteracting the destabilizing force of gravity is usually considered to be the main purpose of postural control. However, from the consideration of the mechanical requirements for movement, we argue that posture is adjusted in view of providing impetus for movement. Thus, we show that the posture that is usually adopted in quiet standing in fact allows torque for potential movement. Moreover, when performing a movement-either voluntarily or in response to an external perturbation-we show that the postural adjustments are organized both spatially and temporally so as to provide the required torque for the movement. Thus, when movement is performed skillfully, the force of gravity is not counteracted but actually used to provide impetus to movement. This ability to move one's weight so as to exploit the torque of gravity seems to be dependent on development and skill learning, and is impaired in aging.

Keywords: neuromechanics, posture, balance, motor control, movement

\section{INTRODUCTION}

\section{OPEN ACCESS}

Edited by:

Matthew Tresch,

Northwestern University, United States

Reviewed by:

Robert H. Lee,

Emory University, United States

Daya Shankar Gupta,

Camden County College,

United States

${ }^{*}$ Correspondence:

Charlotte Le Mouel

charlotte.le-mouel@inserm.fr

Received: 23 February 2017 Accepted: 05 July 2017

Published: 27 July 2017

Citation:

Le Mouel C and Brette R (2017)

Mobility as the Purpose of

Postural Control.

Front. Comput. Neurosci. 11:67.

doi: 10.3389/fncom.2017.00067
The position of the center of mass (CoM) is adjusted by the central nervous system during quiet standing (Winter et al., 1998; Sasagawa et al., 2009), in reaction to perturbations (Horak and Nashner, 1986), and in voluntary movement (Cordo and Nashner, 1982; Pedotti et al., 1989; Lee et al., 1990). The traditional theory is that the purpose of this postural control is to immobilize the CoM despite movement and external perturbations (Nashner et al., 1989; Massion et al., 2004; Horak, 2006; Bouisset and Do, 2008). We will refer to this theory as the immobility theory. The underlying assumption is that, because of gravity, standing is unstable. Therefore, if the $\mathrm{CoM}$ is displaced from its equilibrium position, then the displacement must be counteracted by postural adjustments, so as to return the CoM to its equilibrium position, otherwise the person will inevitably fall. As argued by Hasan (2005), this notion stems from an analysis of how linear systems respond to perturbations: in linear systems, if deviations from the unique equilibrium position are not corrected, then they grow exponentially. Balance (the ability to prevent falling), is therefore assumed to be equivalent to stabilization, in the strict sense of immobilizing the CoM at a unique equilibrium position by counteracting any displacement away from this position. From this assumption, it follows that moving poses a threat to balance, since any voluntary movement might displace the CoM. This theory has motivated a large body of experiments, performed over the last thirty years, in which a subject is asked to perform a movement of the upper body, while their muscle activity is being recorded (Cordo and Nashner, 1982; Crenna et al., 1987; Pedotti et al., 1989; Lee et al., 1990). In these experiments, a change in the contraction of the lower leg muscles is systematically observed, and this change often precedes the contraction of the upper body muscles. This is interpreted by saying that movement of the upper body might displace the CoM, and must therefore be counteracted by the contraction of the lower leg muscles so as to immobilize the CoM despite movement. 
We will argue however that the equivalence between balance and immobilization does not hold for human postural control, and that these postural responses should be understood as providing the impetus for the movement. We will indeed show that during quiet standing, voluntary movement, and in reaction to perturbations, the position of the CoM is not immobilized at a unique equilibrium position, but on the contrary adjusted so as to use the torque of one's own weight, either to counteract external forces so as to maintain balance, or to provide impetus for voluntary movement. We therefore develop an alternative to the immobility theory. We propose that the purpose of postural control is mobility, the ability to produce appropriate impetus by adjusting the position of the CoM. We will refer to this theory as the mobility theory.

We will first show that the posture which is typically adopted in quiet standing allows for one's weight to be used to provide impetus to potential movement, and that when the direction of the movement to be performed can be anticipated, the position of the CoM during stance is shifted in that direction. Secondly, we will show that, during voluntary movement, postural adjustments which are traditionally thought of as immobilizing the CoM despite movement should on the contrary be interpreted as displacing the CoM at the initiation of the movement, so that one's own weight can be used to provide impetus to the movement. Finally, we will show that this ability to use displace one's weight, rather than immobilize it, plays a crucial role when balance is upset by external forces.

\section{ADJUSTMENT OF POSTURE DURING STANCE}

\section{The Standing Posture Allows For Mobility The Standing Posture Requires Tonic Muscular Contraction}

When someone is asked to stand quietly, without further instructions, they typically maintain their CoM vertically aligned with the middle of the foot, a few centimeters forwards of the ankle joint (Schieppati et al., 1994). However, when requested to do so, a young, healthy person can maintain their CoM at positions up to $40 \%$ of their foot length forwards of its typical position, and up to $20 \%$ backwards (Schieppati et al., 1994). There is therefore no unique equilibrium position for the CoM in quiet standing, since a young, healthy person can maintain a range of standing postures without this posing a threat to balance.

If the position of the CoM were controlled only in view of counteracting the torque of one's weight, then it would be most appropriate to place it vertically above the ankles, such that weight would exert no torque (Figure 1A). This position can indeed be maintained with minimum lower leg muscle contraction (Schieppati et al., 1994). However, when no instructions are given, subjects maintain their CoM vertically aligned with the middle of the foot, a few centimeters forwards of the ankle joint (Figure 1B), so that the weight exerts a forwards torque. In order to maintain this posture, an equivalent backwards torque must be exerted by the ground reaction force (see Section 1.1 in Appendix). As developed in the Appendix (Section 1.2), the torque of the ground reaction force is determined by the contraction of the lower leg muscles. Indeed, if we consider the forces acting on the foot, the weight of the body, carried by the skeleton, is applied at the ankle and therefore exerts no torque. The ground prevents the foot from turning, therefore the ground reaction torque instantly opposes the torque exerted by the lower leg muscles onto the foot (Figure 2). Maintaining a standing posture with the CoM forwards of the ankles therefore requires tonic contraction of the calf muscles (Figure 1B, Schieppati et al., 1994). The normal standing posture is therefore not the most economical in terms of muscular contraction.

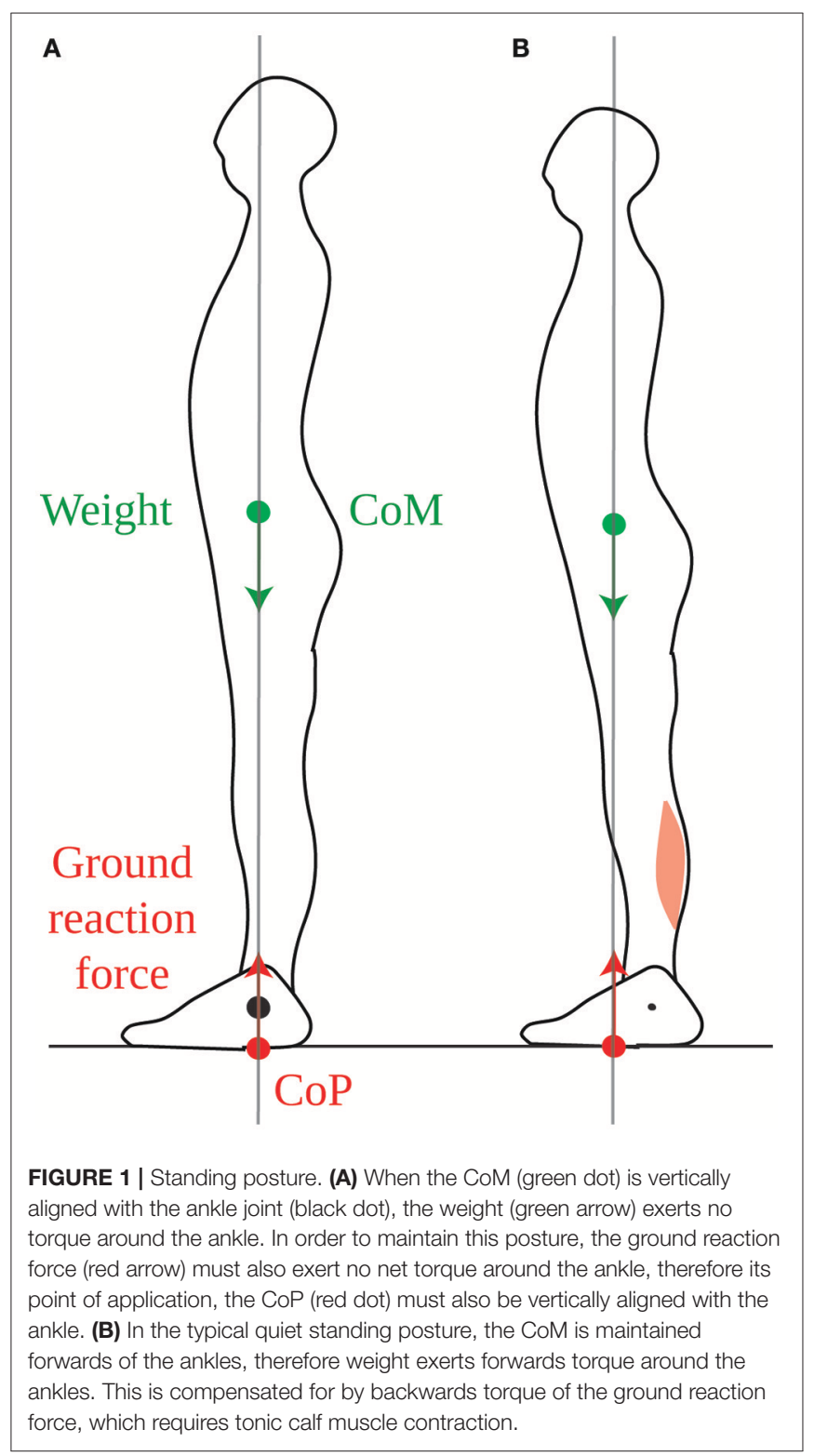




\section{The Standing Posture Allows Torque for Movement}

Why would subjects actively maintain their CoM forwards of the ankles in quiet standing if this is not efficient? We suggest that this allows them to use their own weight for initiating forwards movements. Forwards torque for movement can only be induced by the external forces: the person's weight and the ground reaction force. As we have shown (Appendix Section 1.2) the ground reaction torque instantly follows the torque of the lower leg muscles. However, this torque is limited. Indeed, as long as the person neither jumps up nor collapses, the ground reaction force has the same magnitude as the person's weight. Its torque is therefore the product of the weight, and the distance between the ankle and the point of application of the ground reaction force, called the center of pressure and noted CoP. Thus, contracting the calf muscles (gastrocnemius and soleus) shifts the CoP forwards of the ankle (Figure 2A), and contracting the shin muscle (tibialis anterior) shifts the CoP backwards of the ankle (Figure 2C), but the CoP can only move within the limited range of the foot (see Appendix Section 1.3 for further detail).

The net torque is proportional to the distance between the $\mathrm{CoM}$ and the CoP. Whereas, the CoP moves instantly when the forces exerted by the muscles change, but can only move within the limited range of the foot, the position of the CoM on the other hand, does not change instantly when the forces exerted by the muscles change. This first requires the sum of the external forces to accelerate the CoM. Displacements of the CoM therefore occur more slowly than displacements of the CoP (as seen for example in Burleigh et al., 1994). Thus, the initial

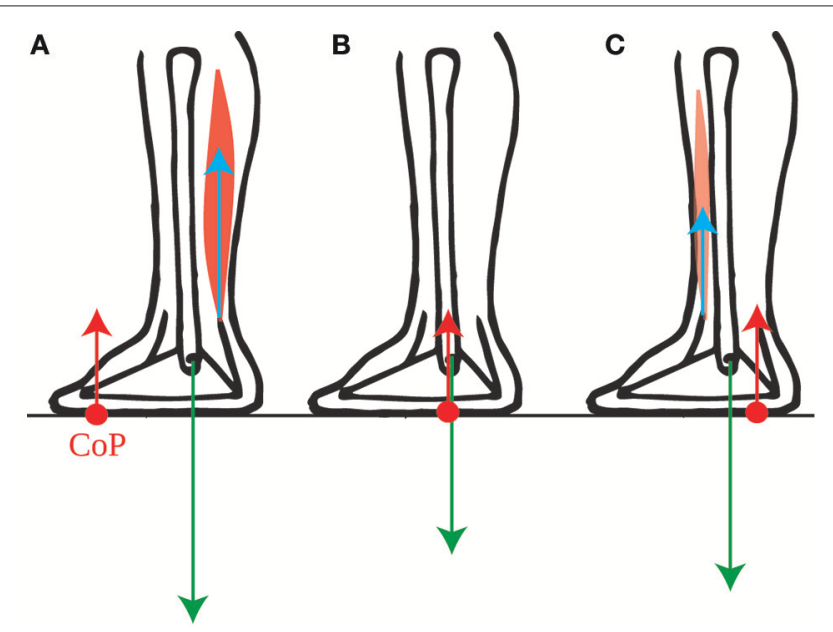

FIGURE 2 | Torques exerted on the foot. The force exerted by the lower leg bones onto the foot (green arrow) exerts no torque around the ankle. The torque of the ground reaction force (red arrow) and of the forces exerted by the lower leg muscles onto the foot (blue arrow) are therefore opposite when the foot remains immobile: (A) the torque around the ankles exerted by the calf muscles onto the foot is instantly compensated for by a forwards shift of the CoP (red dot). (B) When the lower leg muscles exert no torque onto the foot, then the CoP is below the ankle. (C) The torque around the ankles exerted by the shin muscle onto the foot is instantly compensated for by a backwards shift of the CoP. net torque that can be produced, either for opposing external perturbation forces or for voluntary movement, is limited by the initial position of the CoM (see Appendix Section 1.4 for further detail).

When initiating fast forwards movements, either starting to walk (Burleigh et al., 1994) or movements performed with the feet in place such as leaning forwards (Crenna et al., 1987) or rising onto one's toes (Nardone and Schieppati, 1988), the $\mathrm{CoP}$ is first brought toward the heel by inhibiting the calf muscle contraction and contracting the shin muscle (Crenna et al., 1987; Nardone and Schieppati, 1988; Burleigh et al., 1994). If the CoM were initially above the ankle, this would produce little initial forwards torque (Figure 3A), whereas with the CoM forwards of the ankle this produces larger torque (Figure 3B). Maintaining the CoM forwards of the ankle thus allows one's own weight to be used for initiating forwards movement. Maintaining the CoM in the middle of the foot allows for either forwards or backwards initial torque to be induced by changes in the forces of the lower leg muscles.

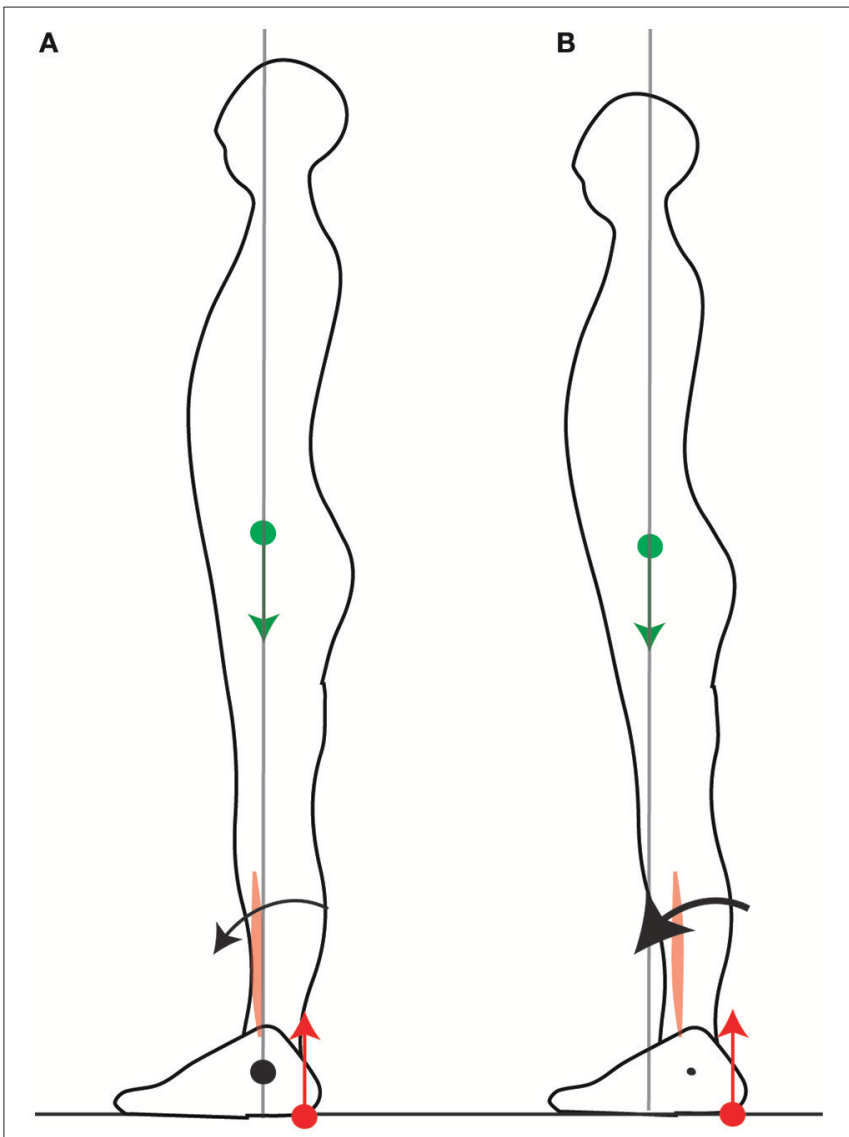

FIGURE 3 | Net torque is limited by the position of the CoM. In order to initiate a forwards movement, the CoP is brought to the heel by inhibiting calf muscle contraction and contracting the shin muscle. When the CoM is vertically aligned with the ankle (A), the net forwards torque is small. When the CoM is forwards of the ankle (B), the net forwards torque is larger. 


\section{The Standing Posture Is Actively Maintained}

This position of the CoM is precisely and actively maintained on a short timescale, with small adjustments of the CoP in quiet standing serving to immobilize the CoM at this position (Winter et al., 1998). Moreover, the tonic contraction of the calf muscles is adjusted when standing on different slopes so as to maintain the CoM aligned with the middle of the foot (Figure 4A, Sasagawa et al., 2009). This precise positioning is also maintained at the longer timescales of growth and aging. Indeed, the curvature of the spine and trunk increases with aging (red line in Figure 4B, Schwab et al., 2006), and the position of the CoM is maintained across people with different trunk curvatures by shifting the position of the pelvis relative to the heels (Figure 4B, Schwab et al., 2006; Lafage et al., 2008).

Moreover, this forwards position of the CoM emerges with skill learning. Thus, Clément and Rézette (1985) observed acrobats at various competitive levels performing handstands. All the acrobats were able to maintain their balance in the upside-down posture, however they did so in different ways. The acrobats at lower competitive levels maintained their mean CoP a few millimeters forwards of their wrist; they could therefore maintain their posture with very little tonic contraction in the arm muscles (Figure 4C, left). The acrobats at higher competitive levels maintained their mean $\mathrm{CoP}$ more forwards of their wrists, with the acrobat at the highest level maintaining his mean CoP 3 $\mathrm{cm}$ forwards of his wrists; this posture requires tonic contraction of the wrist extensors (Figure 4C, right).

Thus, the standing posture is actively adjusted so as maintain the CoM above the middle of the foot (and above the middle of the hand in handstands). Contrary to the immobility theory, this position is not a unique equilibrium point, since a variety of standing postures can be maintained without this leading to a loss of balance. According to the mobility theory, this position is maintained because it allows for torque of the appropriate direction to be produced at short notice, even when this direction cannot be anticipated. This may be useful both for opposing external perturbations and for initiating voluntary movements.

\section{The Standing Posture Is Adjusted in Anticipation of Movement}

When the direction of the appropriate torque can be anticipated, the mobility theory predicts that the CoM would be displaced in that direction in anticipation of the movement. Such a shift can indeed be induced experimentally, either by challenging someone's balance in a predictable direction, or by indicating in advance the direction of a voluntary movement to be performed.

Someone's balance can be challenged by having them stand facing the edge of the platform they are on. According to the immobility theory, this should lead, if anything, to an even more stringent immobilization of the CoM at its equilibrium position,
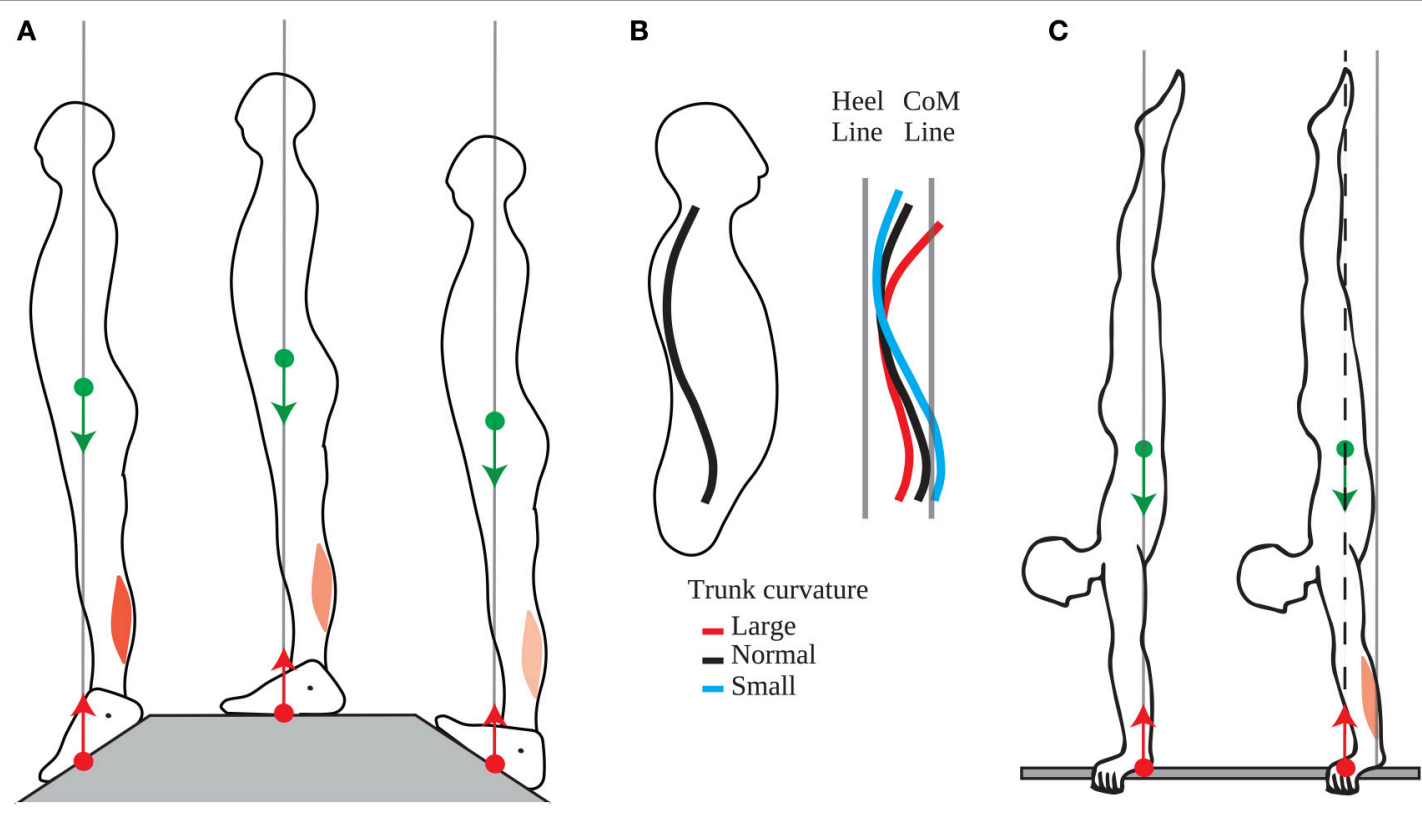

FIGURE 4 | Adaptation of the position of the CoM. (A) The tonic calf muscle contraction decreases when going from a slope with the toes down (left panel), to a flat slope (middle panel), to a slope with the toes up (right panel) such that position of the CoM is maintained vertically aligned with the middle of the feet. (B) People of different trunk curvatures maintain their pelvis at different distances from the heel line (vertical line above the heel), such that the CoM line (vertical line passing through the CoM) is at the same distance from the heel line. (C) Left panel: Acrobats at lower competitive levels maintain their CoP and CoM aligned with their wrist without tonic contraction of their wrist extensors. Right panel: Acrobats at higher competitive levels maintain their CoP and CoM forwards of their wrist, through tonic contraction of their wrist extensors. 
but what is observed is that the CoM is shifted slightly backwards (Figure 5A, Carpenter et al., 2001). This is in accordance with the mobility theory, since it increases the person's capacity for producing backwards torque, in the eventuality that they might be subjected to a forwards push. In the experiment, the person's balance was not challenged beyond placing them in front of a drop, which might explain why the shift in CoM position was rather small (less than a centimeter).

Another way of challenging someone's balance is to have them stand on a platform (Figure 5B) which is then translated backwards (Figure 5C). The person ends up with their CoM in a forward position relative to the feet. A commonly observed response to such a translation is to straighten up (Welch and Ting, 2014). This requires backwards torque, however their capacity for producing backwards torque is limited by the forwards position of their CoM (Figure 5C). If such a perturbation is repeated, then over a few trials, the person adjusts their quiet standing posture by shifting their CoM backwards by a few centimeters (Figure 5D, Welch and Ting, 2014). This is again in contradiction with the immobility theory, but in accordance with the mobility theory, since the backwards shift of the CoM increases the person's capacity to produce backwards torque for straightening up (Figure 5E). When the platform is repeatedly translated forwards, then the person shifts their CoM forwards (Welch and Ting, 2014).

The mobility theory predicts that the position of the CoM in quiet standing would also be shifted if the direction in which a voluntary movement to be performed could be anticipated. This occurs at the start of a race: in sprinting, the initial forwards acceleration is crucial in winning the race. Consistently with the mobility theory, the CoM in the starting position is shifted even beyond the toes by several tens of centimeters (Slawinski et al., 2010). This is achieved by placing the hands on the ground and having the hands carry some of the weight (Figure 5F). This ability to use one's own weight to produce torque for movement again seems to depend on skill learning. Indeed, in elite sprinters, the CoM is shifted 5 centimeters further forwards than for welltrained sprinters (Slawinski et al., 2010).

\section{Summary}

Thus, when the direction of the appropriate torque to be produced cannot be anticipated, the CoM is positioned at the middle of the feet, in a position which allows for both forwards and backwards torque to be produced. When the direction of the torque to be produced can be anticipated, then the standing posture is adjusted by shifting the CoM in that direction. This adaptation of the standing posture in view of movement seems to be dependent on learning.

\section{ADJUSTMENT OF POSTURE DURING VOLUNTARY MOVEMENT}

According to the immobility theory, when a voluntary movement is being performed, postural control serves to immobilize the CoM despite the movement or the perturbation. The mobility theory predicts, on the contrary, that the position of the CoM

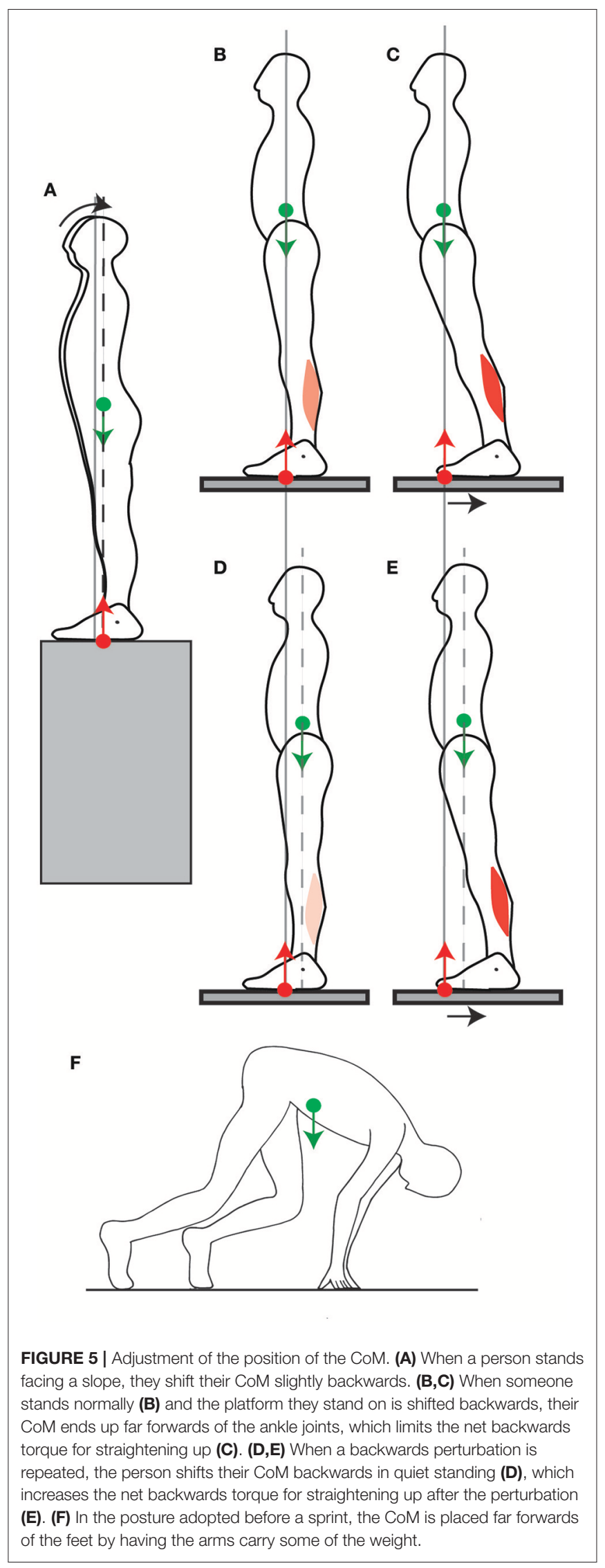


is adjusted so as to use the torque of weight for movement. It therefore predicts that muscular contractions are temporally organized so as to accelerate the CoM at the initiation of the movement in the appropriate direction for producing torque for movement.

\section{Initiation of Voluntary Movement Pulling on a Handle}

When someone pulls on a handle placed in front of them, the contraction of the arm muscles is preceded then accompanied by the contraction of the calf muscles (Cordo and Nashner, 1982; Lee et al., 1990). Cordo and Nashner (1982) suggest that this contraction of the calf muscles allows for the CoM to be immobilized despite the movement. However, in order for the CoM to be immobilized, the ground reaction torque would have to exactly compensate for the handle reaction torque throughout the movement, and this would notably require the calf and arm muscle contractions to be simultaneous (as in Figure 6A). On the contrary, the initial contraction of the calf muscles which is observed (Cordo and Nashner, 1982) accelerates the CoM backwards (Figures 6B,C, further details are provided in Appendix Section 2); and when the person is asked to pull harder on the handle, this initial period lasts longer, the calf muscle activation is stronger, and the initial backwards acceleration of the CoM is larger (Lee et al., 1990). This is in accordance with the mobility theory, since initially accelerating the CoM backwards allows one's own weight to be used to assist the movement (Figure 6C).

\section{Leaning the Trunk}

When someone leans the trunk forwards, the contraction of the abdominal muscles is preceded then accompanied by the inhibition of calf muscle contraction and the contraction of the shin muscle (Figures 7A-C, Crenna et al., 1987). The CoM could in theory be immobilized if the shin and abdominal muscle contractions were simultaneous, such that the forwards acceleration of the CoM induced by the shin muscle contraction would compensate for the backwards acceleration of the CoM induced by the abdominals contraction (further details are provided in Appendix Section 2), as suggested by Alexandrov et al. (2001). However, these authors report an initial backwards displacement of the CoP (Figure 7A), followed by a forwards displacement of the CoM (Figure 7B), in accordance with the sequential muscular contraction observed by Crenna et al. (1987). This contradicts the immobility theory, but concords with the mobility theory's predictions.

Thus, postural responses should be considered as an integral part of the movement itself, since they provide the torque for the movement, first by shifting the CoP and secondly by accelerating the CoM through sequential muscle contraction (a more complete explanation can be found in Appendix Section 2).

\section{Gait Initiation}

Bouisset and Do (2008) distinguish between two types of anticipatory postural adjustments. For voluntary movements without a change in the basis of support, such as raising the arm, they provide a very classical interpretion for the displacement of the CoM which precedes the displacement of the arm. They present it as a counterperturbation whose purpose is to "counterbalance the disturbance to postural equilibrium due to the intentional forthcoming movement" (Bouisset and Zattara, 1981). However, for voluntary movements involving a change of the basis of support, such as walking, or rising onto one's toes,

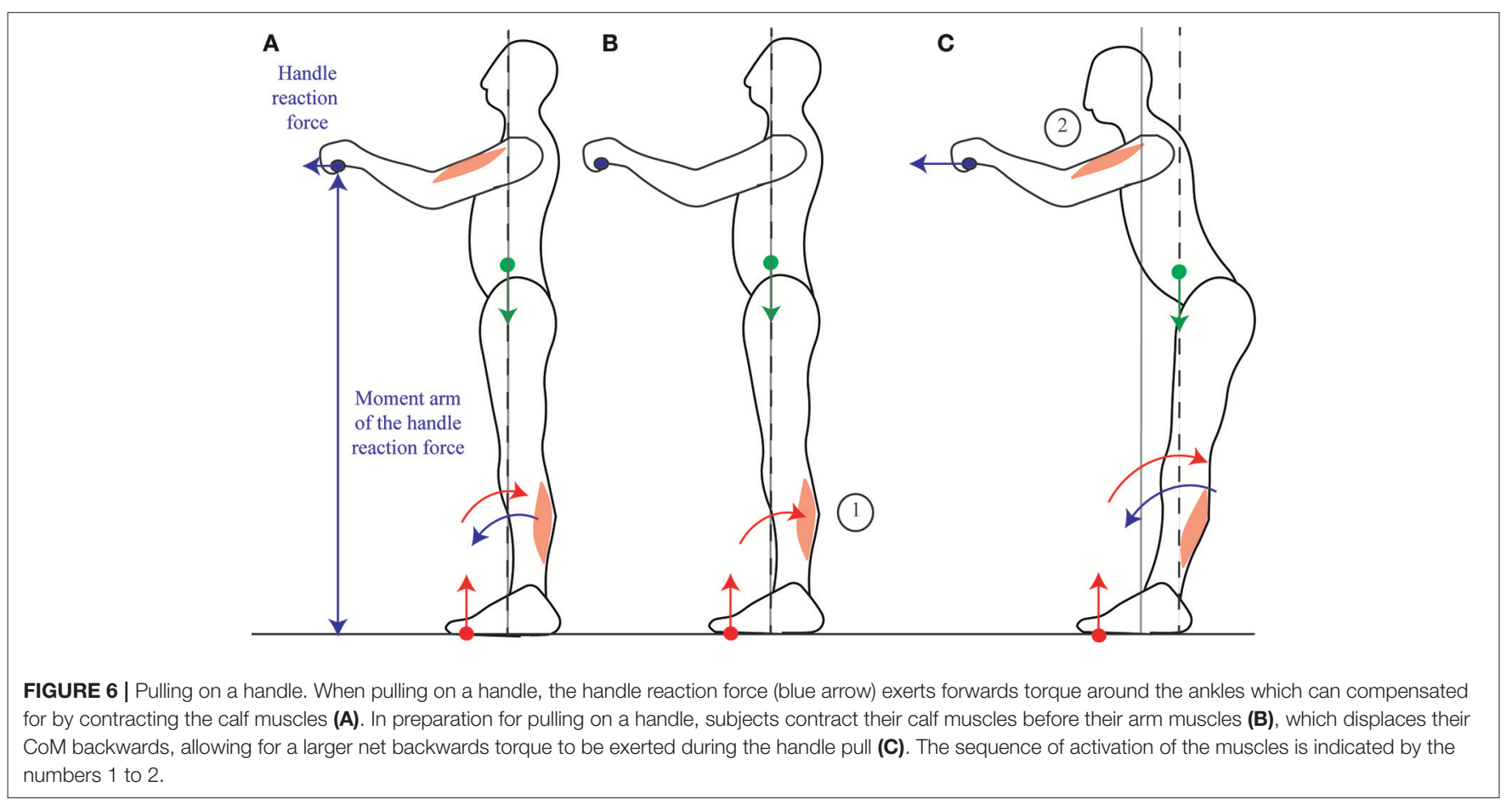




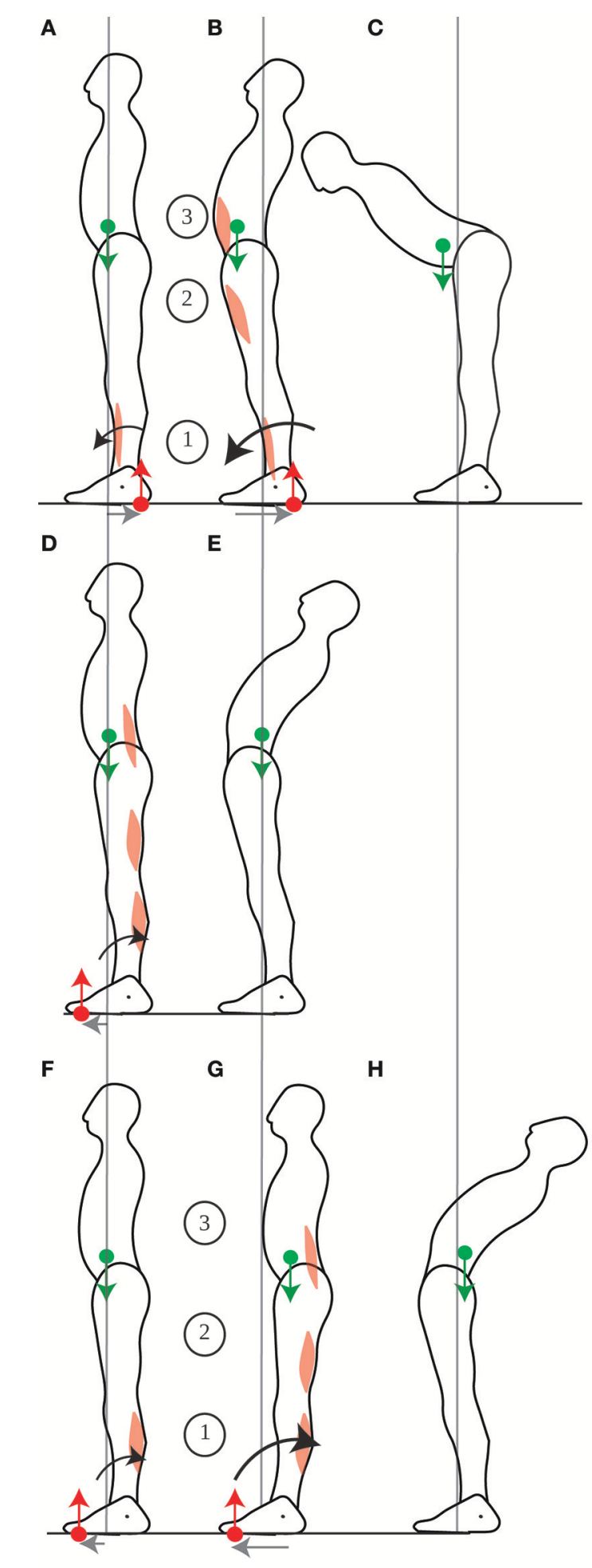

FIGURE 7 | Leaning the trunk. When control subjects perform fast forwards leaning, the initial contraction of the shin muscle (A) accelerates the CoM forwards, thus allowing for more net forwards torque during the subsequent contraction of the ventral muscles (B), which enables the person to lean the trunk (C). When control subjects perform fast backwards leaning, the dorsal muscles contract simultaneously (D), which increases backwards rotational

(Continued)
FIGURE 7 | Continued

momentum without translating the CoM (E). When gymnasts perform fast backwards leaning, the initial contraction of the calf muscles $(\mathbf{F})$ accelerates the CoM backwards, thus allowing for more net backwards torque during the subsequent contraction of the dorsal muscles (G) which enables the gymnast to lean the trunk $\mathbf{( H )}$. The sequence of activation of the muscles is indicated by the numbers 1 to 3 .

they present anticipatory postural adjustments as a perturbation involved in "body weight transfer" (Do et al., 1991).

We propose that in movements with or without a change in the basis of support, anticipatory postural adjustments play the same role of moving the CoM in order to provide impetus for movement. Indeed, the changes in posture which precede walking are organized in the same way as those which precede pulling on a handle or leaning the trunk. Thus, when going from standing to walking, a few hundred milliseconds before the heel of the swing foot is raised, the calf muscles are silenced and the shin muscle contracts, which brings the CoP to the heels and accelerates the CoM forwards, even before the first step is taken (Figure 3B, Burleigh et al., 1994). This is in accordance with the mobility theory, since initially accelerating the CoM forwards allows one's own weight to be used to assist the movement. Indeed, this initial acceleration of the CoM is correlated with the speed reached at the end of the first step, and is larger if the person is asked to walk faster (Brenière et al., 1987).

\section{The Ability to Use One's Weight for Movement Requires Practice}

For walking, a movement which is learned very early on in life, the ability to displace the CoM at the initiation of the movement emerges over the course of development (Ledebt et al., 1998; Bril et al., 2015). The amplitude of the initial backwards shift of the $\mathrm{CoP}$ thus increases over the first several years of life as children learn to walk faster (Ledebt et al., 1998; Bril et al., 2015). It then decreases with age, and with certain neurological diseases such as Parkinson's disease (Halliday et al., 1998; Mancini et al., 2016).

For leaning the trunk, the sequential muscle contraction, which allows for the displacement of the CoM at the initiation of the movement, seems to be dependent on learning. Indeed, when control subjects are asked to lean backwards, a movement for which they presumably have less practice than leaning forwards, then the calf and dorsal trunk muscle contractions are simultaneous (Figure 7D), and the movement is performed twice as slowly as leaning forwards (Pedotti et al., 1989). This is presumably because the CoM was not displaced backwards (Figure 7E). However, when gymnasts are asked to lean backwards, then their calf muscles contract first, and they perform the movement faster than controls (Figures $7 \mathbf{F}-\mathbf{H}$, Pedotti et al., 1989). Moreover, the ability to displace one's CoM during movement seems to remain plastic throughout life, and to depend on the possibility to use one's weight to assist movement. Thus, when astronauts return from a several months journey in space (during which they could not use their weight to assist 
their movements), the forwards displacement of the CoM when leaning forwards is no longer observed (Baroni et al., 2001).

Finally, for movements requiring skill learning, the temporal coordination which enables using one's weight to provide impetus for movement seems to develop with skill learning. Thus, when learning a complex gymnastics skill, such as the swings under parallel bars, in bent inverted hang position (Figure 8), beginners swing their legs and arms in synchrony, whereas experts swing their legs out of phase with their arms, which allows them to use the work of their own weight to provide impetus to the swing (Delignières et al., 1998).

\section{BALANCE REQUIRES MOBILITY RATHER THAN IMMOBILITY}

According to the immobility theory, if postural control does not immobilize the CoM at a unique equilibrium position, then the person must fall (Nashner et al., 1989; Massion et al., 2004; Horak, 2006; Bouisset and Do, 2008). We have shown however that in quiet standing, people can keep their balance over a range of positions of the CoM (Schieppati et al., 1994), and actually displace their CoM when their balance is challenged in a predictable (Carpenter et al., 2001; Welch and Ting, 2014). Moreover, we have shown that in well-practiced movements, people accelerate their CoM at the initiation of the movement, without this leading to a loss of balance (Cordo and Nashner, 1982; Crenna et al., 1987; Pedotti et al., 1989; Lee et al., 1990). We will now show that the response to an external perturbation should be considered as a movement in its own right, and therefore also benefits from the ability to use one's weight for movement, rather than to immobilize it.

\section{Responding to External Perturbations Straightening Up after a Platform Translation}

When the platform on which someone stands is translated backwards, the CoM ends up in a forward position relative to the feet (Figures 9A,B), as seen in Section The standing posture is adjusted in anticipation of movement. A response which is commonly observed is to straighten up (Horak and Nashner, 1986). The backwards acceleration of the CoM is performed through a sequential contraction of the dorsal muscles, starting with the calf muscles (Figure 9A), then the dorsal thigh then dorsal trunk muscles (Horak and Nashner, 1986). This contraction pattern is usually not considered as an actual movement, since it moves the CoM closer to its initial position, in accordance with the immobility theory. However, we believe it should be considered as a movement in its own right. Indeed, straightening up after a platform translation requires producing the appropriate backwards torque. The sequential contraction pattern allows for the CoM to be initially accelerated backwards, which increases the net backwards torque for the movement. Further details are provided in Appendix-Horizontal acceleration of the CoM. Moreover, contrary to the immobility theory, returning the CoM to its initial position is not the only way of preventing a fall.

\section{Stepping after a Platform Translation}

Indeed, another response which is also commonly observed is to take a step forwards (Maki et al., 2003): the CoM is then not returned to its initial position, without this causing a loss of balance. This response takes advantage of the forwards position of the CoM, such that the CoM needs not be accelerated backwards, and indeed the initial calf muscle contraction and forwards CoP shift is much reduced (Figure 9B) compared to when the person straightens up (Figure 9A); nor does the CoM need to be accelerated forwards, and indeed the shin muscle contraction lasts much less long and the backwards shift of the CoP is much smaller (Figure 9C, Burleigh et al., 1994) than when the person takes a step without the platform translation (Figure 9D).

\section{Emergence Over Development and Impairment with Aging}

The ability to mobilize one's weight emerges over development. Thus, when straightening up after a backwards platform translation, both the systematic recruitment of the dorsal muscles and their temporal sequencing emerge during development. They are not observed in pre-walking infants, but are seen in children with a few years' walking experience (Burtner et al., 1998).

This ability is then deteriorated with aging, and with Parkinson's disease. The elderly, and even more so Parkinsonian patients, are less capable of moving their CoM, either when asked to adjust their quiet standing posture by leaning forwards or backwards (Schieppati et al., 1994), or during voluntary movement, such as gait initiation (Halliday et al., 1998). They are however quite as capable as young healthy adults of remaining immobile in quiet standing (Schieppati et al., 1994), and adjust the position of their pelvis to compensate for trunk curvature

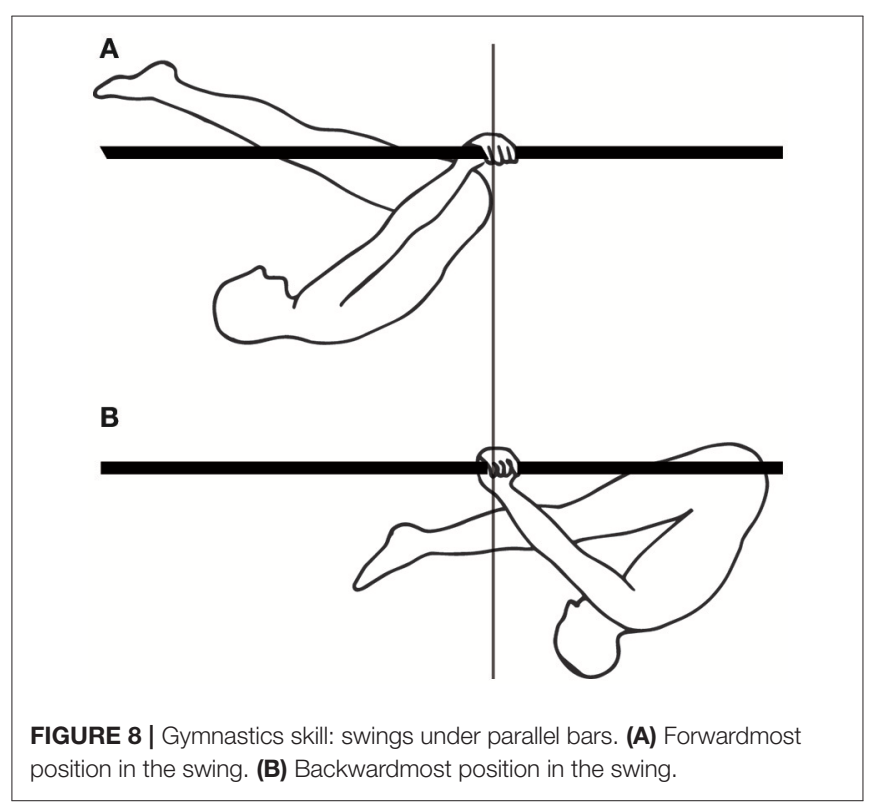


such that the CoM remains above the middle of the feet (Schwab et al., 2006). Nevertheless, they have a heightened risk of falling. Thus, although the elderly and Parkinsonian subjects are quite as capable as young adults of maintaining their CoM immobile during quiet standing, we suggest that their higher risk of falling is due to a limited capacity to move when this becomes necessary to prevent a fall. Therefore, not only is immobilizing the CoM unnecessary for balance, it moreover seems that balance benefits from the ability to move one's CoM. This suggests that efficient balance training for the elderly can be achieved by practicing mobility (Xu et al., 2005).

\section{DISCUSSION}

\section{Posture Is Adjusted in View of Mobility Rather than Immobility}

Although the position of the CoM is adjusted by the nervous system, this postural control does not serve to immobilize the CoM. On the contrary, the position of the CoM is adjusted so as to use the torque of one's own weight both for selfinitiated movements and for responding to external perturbation forces.

Thus, in quiet standing, when the direction of the torque to be produced cannot be anticipated, the CoM is maintained above the middle of the foot (Schieppati et al., 1994), allowing for the torque of one's weight to be used both for forwards and backwards movements. This position is actively maintained despite short-term changes in slope (Sasagawa et al., 2009) or long-term changes in trunk curvature (Schwab et al., 2006). However, when the direction of the torque to be produced can be anticipated, then the CoM is shifted in that direction. There is thus a small backwards shift of the CoM when someone is placed in front of a drop (Carpenter et al., 2001), or on a platform which is repeatedly translated backwards (Welch and Ting, 2014). Skill learning leads to much larger shifts in the position of the CoM, with the CoM placed forwards of the feet in anticipation of sprinting (Slawinski et al., 2010).

Moreover, during movement, we have shown that the postural responses which were thought to immobilize the CoM despite movement are actually temporally organized so as to accelerate the CoM at the initiation of the movement, in the appropriate direction such that the torque of one's weight can be used for the movement (Cordo and Nashner, 1982; Crenna et al., 1987; Pedotti et al., 1989; Lee et al., 1990). These postural responses should therefore be understood as providing impetus to the movement.

Finally, we have shown that in order to respond effectively to external perturbation forces, the CoM need not be immobilized, since the person can take a step (Maki et al., 2003). When the person straightens up without taking a step (Horak and Nashner, 1986), this requires producing forces to counteract the external perturbation, and may benefit from the ability to mobilize one's CoM rather than immobilize it. Balance therefore requires mobility rather than immobility.
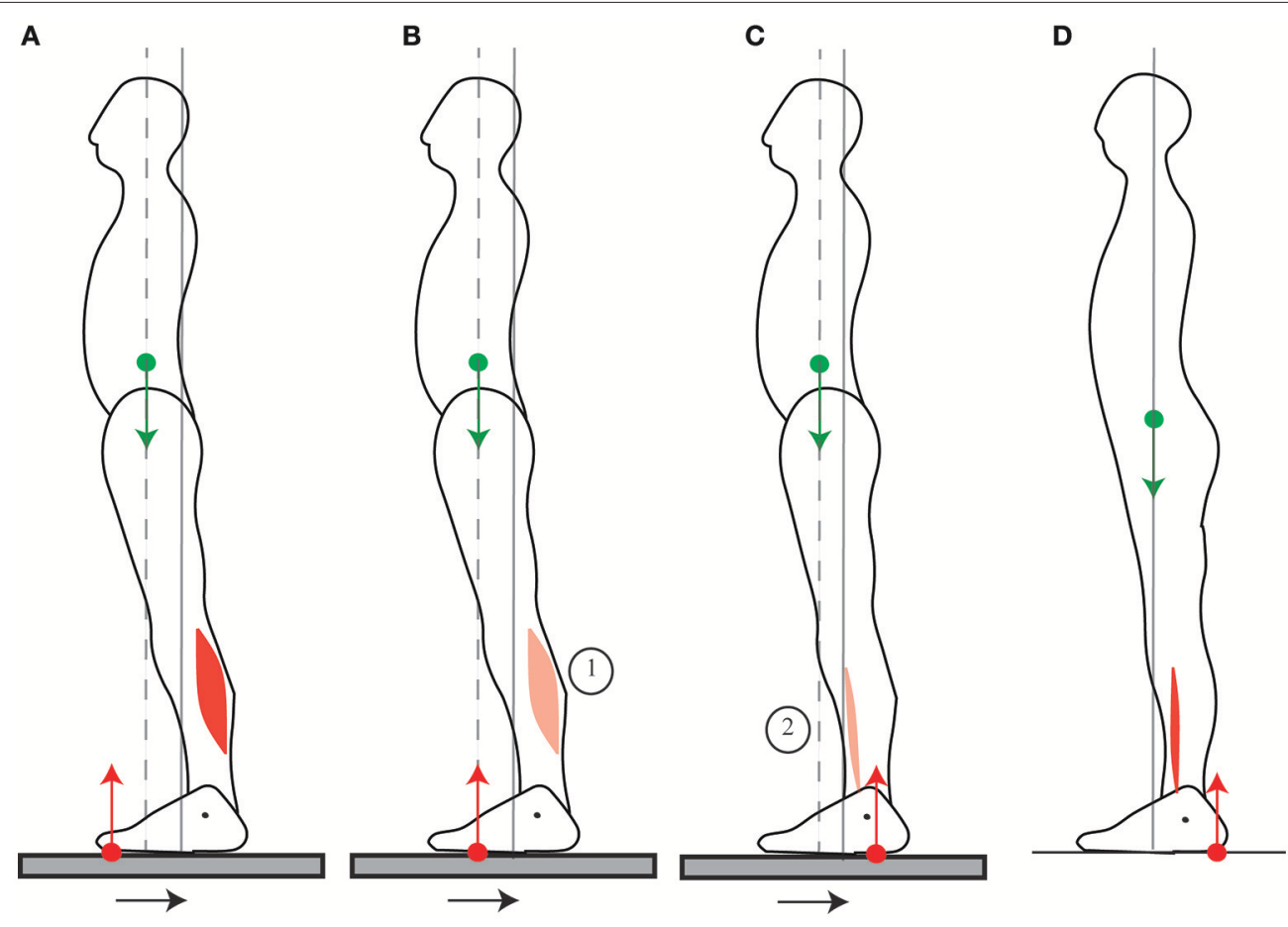

FIGURE 9 | Response to platform translation. When straightening up after a platform translation (A), the initial contraction of the calf muscle accelerates the CoM backwards which increases the potential net backwards torque. When stepping forwards in response to a platform translation (B,C), this initial calf muscle contraction is reduced $\mathbf{( B )}$. Then, the shin muscle contracts $\mathbf{( C )}$. This shin muscle contraction is smaller than the initial contraction of the shin muscle which accelerates the CoM forwards when the person steps forwards without a platform translation (D). The sequence of activation of the muscles is indicated by the numbers 1 to 2. 


\section{Mobility Emerges Through Development and Skill Learning}

The ability to use one's weight for movement emerges through development and skill learning, and remains plastic throughout life. The appropriate temporal organization of muscular contraction emerges during development both for walking and for balancing responses (Burtner et al., 1998; Ledebt et al., 1998). It is not observed for less practiced movements, such as when control subjects lean the trunk backwards (Crenna et al., 1987). The extent to which the CoM can be mobilized seems to depend on the level of skill: thus, both for sprinters at the initiation of a race (Slawinski et al., 2010) and acrobats performing handstands (Clément and Rézette, 1985), elite athletes place their CoM further forwards than well-trained athletes. Future work should address the following questions: how is this ability learned through development and practice? Does the impairment of this ability in aging result from a lack of practice, and could this

\section{REFERENCES}

Alexandrov, A. V., Frolov, A. A., and Massion, J. (2001). Biomechanical analysis of movement strategies in human forward trunk bending. I. Modeling. Biol. Cybern. 84, 425-434. doi: 10.1007/PL00007986

Baroni, G., Pedrocchi, A., Ferrigno, G., Massion, J., and Pedotti, A. (2001). Static and dynamic postural control in long-term microgravity: evidence of a dual adaptation. J. Appl. Physiol. 1985, 205-215.

Bouisset, S., and Do, M.-C. (2008). Posture, dynamic stability, and voluntary movement. Neurophysiol. Clin. Neurophysiol. 38, 345-362. doi: 10.1016/j.neucli.2008.10.001

Bouisset, S., and Zattara, M. (1981). A sequence of postural movements precedes voluntary movement. Neurosci. Lett. 22, 263-270. doi: 10.1016/0304-3940(81)90117-8

Brenière, Y., Cuong Do, M., and Bouisset, S. (1987). Are dynamic phenomena prior to stepping essential to walking? J. Mot. Behav. 19, 62-76. doi: 10.1080/00222895.1987.10735400

Bril, B., Dupuy, L., Dietrich, G., and Corbetta, D. (2015). Learning to tune the antero-posterior propulsive forces during walking: a necessary skill for mastering upright locomotion in toddlers. Exp. Brain Res. 233, 2903-2912. doi: 10.1007/s00221-015-4378-6

Burleigh, A. L., Horak, F. B., and Malouin, F. (1994). Modification of postural responses and step initiation: evidence for goal-directed postural interactions. J. Neurophysiol. 72, 2892-2902.

Burtner, P. A., Qualls, C., and Woollacott, M. H. (1998). Muscle activation characteristics of stance balance control in children with spastic cerebral palsy. Gait Posture 8, 163-174. doi: 10.1016/S0966-6362(98)00032-0

Carpenter, M. G., Frank, J. S., Silcher, C. P., and Peysar, G. W. (2001). The influence of postural threat on the control of upright stance. Exp. Brain Res. 138, 210-218. doi: $10.1007 / \mathrm{s} 002210100681$

Clément, G., and Rézette, D. (1985). Motor behavior underlying the control of an upside-down vertical posture. Exp. Brain Res. 59, 478-484. doi: 10.1007/BF00261337

Cordo, P. J., and Nashner, L. M. (1982). Properties of postural adjustments associated with rapid arm movements. J. Neurophysiol. 47, 287-302.

Crenna, P., Frigo, C., Massion, J., and Pedotti, A. (1987). Forward and backward axial synergies in man. Exp. Brain Res. 65, 538-548. doi: 10.1007/BF00235977

Delignières, D., Nourrit, D., Sioud, R., Leroyer, P., Zattara, M., and Micaleff, J.-P. (1998). Preferred coordination modes in the first steps of the learning of a complex gymnastics skill. Hum. Mov. Sci. 17, 221-241. doi: 10.1016/S0167-9457(97)00031-6

Do, M. C., Nouillot, P., and Bouisset, S. (1991). Is balance or posture at the end of a voluntary movement programmed? Neurosci. Lett. 130, 9-11. doi: 10.1016/0304-3940(91)90215-F ability be maintained during aging through appropriate training regimes?

\section{AUTHOR CONTRIBUTIONS}

All authors listed have made a substantial, direct and intellectual contribution to the work, and approved it for publication.

\section{FUNDING}

This work was supported by Agence Nationale de la Recherche (ANR-14-CE13-0003).

\section{SUPPLEMENTARY MATERIAL}

The Supplementary Material for this article can be found online at: http://journal.frontiersin.org/article/10.3389/fncom. 2017.00067/full\#supplementary-material

Halliday, S. E., Winter, D. A., Frank, J. S., Patla, A. E., and Prince, F. (1998). The initiation of gait in young, elderly, and Parkinson's disease subjects. Gait Posture 8, 8-14. doi: 10.1016/S0966-6362(98)00020-4

Hasan, Z. (2005). The human motor control system's response to mechanical perturbation: should it, can it, and does it ensure stability? J. Mot. Behav. 37, 484-493. doi: 10.3200/JMBR.37.6.484-493

Horak, F. B. (2006). Postural orientation and equilibrium: what do we need to know about neural control of balance to prevent falls? Age Ageing 35 (Suppl. 2), ii7-ii11. doi: 10.1093/ageing/afl077

Horak, F. B., and Nashner, L. M. (1986). Central programming of postural movements: adaptation to altered support-surface configurations. J. Neurophysiol. 55, 1369-1381.

Lafage, V., Schwab, F., Skalli, W., Hawkinson, N., Gagey, P.-M., Ondra, S., et al. (2008). Standing balance and sagittal plane spinal deformity: analysis of spinopelvic and gravity line parameters. Spine 33, 1572-1578. doi: 10.1097/BRS.0b013e31817886a2

Ledebt, A., Bril, B., and Brenière, Y. (1998). The build-up of anticipatory behaviour. An analysis of the development of gait initiation in children. Exp. Brain Res. 120, 9-17. doi: 10.1007/s002210050372

Lee, W. A., Michaels, C. F., and Pai, Y. C. (1990). The organization of torque and EMG activity during bilateral handle pulls by standing humans. Exp. Brain Res. 82, 304-314. doi: 10.1007/BF00231250

Maki, B. E., McIlroy, W. E., and Fernie, G. R. (2003). Change-in-support reactions for balance recovery. IEEE Eng. Med. Biol. Mag. Q. Mag. Eng. Med. Biol. Soc. 22, 20-26. doi: 10.1109/MEMB.2003.1195691

Mancini, M., Chiari, L., Holmstrom, L., Salarian, A., and Horak, F. B. (2016). Validity and reliability of an IMU-based method to detect APAs prior to gait initiation. Gait Posture 43, 125-131. doi: 10.1016/j.gaitpost.2015.0 8.015

Massion, J., Alexandrov, A., and Frolov, A. (2004). Why and how are posture and movement coordinated? Prog. Brain Res. 143, 13-27. doi: 10.1016/S0079-6123(03)43002-1

Nardone, A., and Schieppati, M. (1988). Postural adjustments associated with voluntary contraction of leg muscles in standing man. Exp. Brain Res. 69, 469-480. doi: 10.1007/BF00247301

Nashner, L. M., Shupert, C. L., Horak, F. B., and Black, F. O. (1989). Organization of posture controls: an analysis of sensory and mechanical constraints. Prog. Brain Res. 80, 411-418. Discussion: 395-397. doi: 10.1016/s0079-6123(08)62 237-2

Pedotti, A., Crenna, P., Deat, A., Frigo, C., and Massion, J. (1989). Postural synergies in axial movements: short and long-term adaptation. Exp. Brain Res. 74, 3-10. doi: 10.1007/BF00248275

Sasagawa, S., Ushiyama, J., Masani, K., Kouzaki, M., and Kanehisa, H. (2009). Balance control under different passive contributions of the ankle 
extensors: quiet standing on inclined surfaces. Exp. Brain Res. 196, 537-544. doi: 10.1007/s00221-009-1876-4

Schieppati, M., Hugon, M., Grasso, M., Nardone, A., and Galante, M. (1994). The limits of equilibrium in young and elderly normal subjects and in parkinsonians. Electroencephalogr. Clin. Neurophysiol. 93, 286-298. doi: 10.1016/0168-5597(94)90031-0

Schwab, F., Lafage, V., Boyce, R., Skalli, W., and Farcy, J.-P. (2006). Gravity line analysis in adult volunteers: age-related correlation with spinal parameters, pelvic parameters, and foot position. Spine 31, E959-E967. doi: 10.1097/01.brs.0000248126.96737.0f

Slawinski, J., Bonnefoy, A., Levêque, J.-M., Ontanon, G., Riquet, A., Dumas, R., et al. (2010). Kinematic and kinetic comparisons of elite and welltrained sprinters during sprint start. J. Strength Cond. Res. 24, 896-905. doi: 10.1519/JSC.0b013e3181ad3448

Welch, T. D. J., and Ting, L. H. (2014). Mechanisms of motor adaptation in reactive balance control. PLOS ONE 9:e96440. doi: 10.1371/journal.pone.00 96440
Winter, D. A., Patla, A. E., Prince, F., Ishac, M., and Gielo-Perczak, K. (1998). Stiffness control of balance in quiet standing. J. Neurophysiol. 80, 1211-1221.

Xu, D.-Q., Li, J.-X., and Hong, Y. (2005). Effect of regular Tai Chi and jogging exercise on neuromuscular reaction in older people. Age Ageing 34, 439-444. doi: 10.1093/ageing/afil14

Conflict of Interest Statement: The authors declare that the research was conducted in the absence of any commercial or financial relationships that could be construed as a potential conflict of interest.

Copyright (c) 2017 Le Mouel and Brette. This is an open-access article distributed under the terms of the Creative Commons Attribution License (CC BY). The use, distribution or reproduction in other forums is permitted, provided the original author(s) or licensor are credited and that the original publication in this journal is cited, in accordance with accepted academic practice. No use, distribution or reproduction is permitted which does not comply with these terms. 